\title{
High frequency of mutation G377S in Brazilian type 3 Gaucher disease patients
}

\section{R. Rozenberg1', F.T. Araújo ${ }^{1}$, D.C. Fox ${ }^{1}$, P. Aranda ${ }^{2}$, A. Nonino ${ }^{3}$, C. Micheletti ${ }^{4}$, A.M. Martins ${ }^{4}$, R. Cravo ${ }^{5}$, \\ E. Sobreira ${ }^{6}$ and \\ L.V. Pereira ${ }^{1}$}

\author{
'Departamento de Genética e Biologia Evolutiva, Instituto de Biociências, \\ Universidade de São Paulo, São Paulo, SP, Brasil \\ ${ }^{2}$ Hospital Evangélico de Londrina, Londrina, PR, Brasil \\ ${ }^{3}$ Hospital de Base do Distrito Federal, Brasília, DF, Brasil \\ ${ }^{4}$ Departamento de Pediatria, Escola Paulista de Medicina, \\ Universidade Federal do Estado de São Paulo, São Paulo, SP, Brasil \\ ${ }^{5}$ Serviço de Hemoterapia, HEMORIO, Rio de Janeiro, RJ, Brasil \\ ${ }^{6}$ Serviço de Hemato-Oncologia, Faculdade de Ciências Médicas, \\ Santa Casa de São Paulo, São Paulo, SP, Brasil
}

\section{Correspondence \\ R. Rozenberg \\ Departamento de Genética e \\ Biologia Evolutiva \\ Instituto de Biociências, USP \\ R. do Matão, 277, Sala 350 \\ 05508-900 São Paulo, SP \\ Brasil \\ Fax: +55-11-30917583 \\ E-mail: robertor@usp.br}

Research supported by FAPESP,

CNPq, and Genzyme do Brasil.

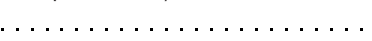

Received December 13, 2005

Accepted July 20, 2006

\begin{abstract}
Gaucher disease (GD), the most prevalent lysosome storage disorder, presents an autosomal recessive mode of inheritance. It is a paradigm for therapeutic intervention in medical genetics due to the existence of effective enzyme replacement therapy. We report here the analysis of GD in 262 unrelated Brazilian patients, carried out in order to establish the frequency of the most common mutations and to provide prognostic information based on genotype-phenotype correlations. Among 247 type 1 GD patients, mutation N370S was detected in $47 \%$ of all the alleles, but N370S/N370S homozygosity was found in only $10 \%$ of the patients, a much lower frequency than expected, suggesting that most individuals presenting this genotype may not receive medical attention. Recombinant alleles were detected at a high frequency: $44 \%$ of the chromosomes bearing mutation L444P had other mutations derived from the pseudogene sequence, present in $25 \%$ of patients. Three neuronopathic type 2 patients were homozygous for L444P, all presenting additional mutations (E326K or recombinant alleles) that probably lead to the more severe phenotypes. Six children, classified as type 1 GD patients, had a L444P/L444P genotype, showing that neuronopathic symptoms may only manifest later in life. This would indicate the need for a higher treatment dose during enzyme replacement therapy. Finally, mutation G377S was present in 4 homozygous type 1 patients and also in compound heterozygosity in 5 (42\%) type 3 patients. These findings indicate that G377S cannot be unambiguously classified as mild and suggest an allele-dose effect for this mutation.
\end{abstract}

\section{Introduction}

Gaucher disease (GD), the most prevalent lysosome storage disorder, presents an autosomal recessive mode of inheritance.
Key words

- Gaucher disease

- GBA gene

- Allele dose-effect

......................
Clinically, it is a multisystemic disease with variable expression, generally characterized by hepatosplenomegaly, anemia and thrombocytopenia. Bone and pulmonary disease may also be present. The presence and sever- 
ity of neurologic symptoms define the three types of GD. Approximately $95 \%$ of patients present type 1 GD (MIM\# 230800), without neurologic involvement and with onset during childhood or adulthood (1). Neuronopathic variants are type 2 GD (MIM\# 230900), characterized by early onset and survival of up to 2 years of age, and type 3 GD (MIM\# 231000) of infantile or juvenile onset and with a less ominous course with survival into adulthood.

GD is caused by mutations in the GBA gene (1q21). This is a 7-kb gene containing 11 exons, which codes for the acid B-glucosidase enzyme, also known as glucocerebrosidase (2). The absence of this enzyme leads to the accumulation of glucocerebroside in the cells of the reticuloendothelial system and ultimately to the clinical manifestation of GD. More than 200 GD-causing mutations have been identified so far and the most frequent ones are [c.1226A $>$ G;N370S] and [c.1448T $>C ; L 444 P]$, herein called N370S and L444P, respectively. The clinical diagnosis of GD may be confirmed by the biochemical assay of acid ß-glucosidase activity. Unlike enzymatic diagnosis, mutation analysis has a considerable, although not absolute, predictive value with respect to disease prognosis (1). Opportunely, an extensive genotype-phenotype correlation is present for the most frequent mutations. Generally, GD-causing mutations are classified as mild, severe or null alleles (3). The presence of a mild mutation leads to type 1 GD even in combination with a second severe mutation or a null allele. The neuronopathic variants are due to homozygosity of severe mutations, or the combination of a severe mutation and a null allele. Homozygosity of null alleles is not found, suggesting that this genotype is incompatible with life. Despite the genotype-phenotype correlation, a variable expression of clinical symptoms is present in patients with the same genotype, indicating the existence of other factors, genetic and/or environmental, influ- encing the phenotype (4).

GD is the first lysosome storage disease for which an efficient enzyme replacement therapy (ERT) was developed (Imiglucerase) and became a paradigm for therapeutic intervention in human genetics (5). ERT is the standard treatment for type 1 and 3 GD patients, and efficiently ameliorates non-neurological symptoms. The enzyme dose infused during ERT is established for each patient according to body weight and clinical manifestations. Dose individualization is subject to medical decision including the initial dose and its reduction during the lifelong treatment. The high cost of treatment dictates that ERT should be administered at the most efficient possible dose. In Brazil, the patients' therapy is subsidized by the public health system. Thus, the disease may be of low incidence but has a significant economic impact. There are no estimates of GD prevalence in Brazil, but there are approximately 400 diagnosed cases (6), the third largest patient population undergoing ERT in the world.

In this study the frequency of the most common mutations in a sample of 262 unrelated Brazilian GD patients was determined. Several genotype-phenotype correlations were identified and their implications for the diagnosis and treatment of GD are discussed.

\section{Patients and Methods}

\section{Gaucher disease patients and relatives}

Overall, 580 samples were received in the laboratory, from patients, relatives and cases with an unconfirmed clinical diagnosis of GD. Samples came from the different regions of Brazil during a three-year period (2000-2003). The patients with confirmed GD were diagnosed by their doctors after medical evaluation and the determination of reduced glucocerebrosidase activity measured in leukocytes (6). Only three patients were Ashkenazi Jewish. Mouth mucus was 
collected by physicians or health professionals participating in the treatment of the patients and sent to the laboratory in 2-mL Eppendorf tubes. Participants signed informed consent forms and the project was approved by the Brazilian National Committee of Ethics in Research (CONEP).

Mutation screening, for statistical purposes was performed in one case from each family, for a total of 262 cases, 247 of which were classified by their physicians as type 1 , 3 as type 2, and 12 as type 3 GD patients. Since not all of these patients were evaluated by one of the authors, we could not confirm the classification in every case. Most of these patients are undergoing ERT, except type 2 cases, which are not treated and have a reduced life expectancy. The genotype was analyzed separately for type 2 and 3 patients in order to avoid bias in the evaluation of allelic and genotypic frequencies.

\section{Molecular diagnosis}

DNA was extracted from mouth mucus by the method of Richards et al. (7). Mutations N370S, L444P, G377S, c.84insG (84insG), IVS2+1G>A, c.1263_1317del (55del), V460V, D409H, and E326K were analyzed on the basis of restriction fragment length polymorphism. The enzymatic effect of these mutations, their screening strategy, the primers (Invitrogen, Carlsbad, CA, USA), and restriction endonucleases (New England Biolabs, Ipswich, MA, USA or from Fermentas, Vilnius, Lithuania) used for detection and the reference method, when previously established, are described in Table 1. After endonuclease digestion, fragments were submitted to $12 \%$ polyacrylamide gel electrophoresis (mutations N370S, G377S, 84 insG, and IVS $2+1 \mathrm{G}>\mathrm{A}$ ) or $2 \%$ agarose gel electrophoresis (mutations L444P, V460V, D409H, 55del, and E326K).

All patients were screened for mutations N370S and L444P, the most frequent mutations worldwide. Mutation G377S is the third most frequent mutation in Portugal and was therefore also included in the initial analysis. In the absence of these three mutations in one of the alleles, the first one hundred patients were screened for mutations 84insG and IVS2+1G $>$ A. Patients homozygous for N370S, G377S or L444P were investigated for the presence of mutation 55del, present in the pseudogene (psGBA) contiguous to the GBA gene. This deletion, if present in GBA, would be localized at the annealing site of primers used for detecting mutations N370S, G377S and L444P (8).

Because mutation L444P may be a part of a recombinant allele (REC), with DNA sequence derived from the highly homologous psGBA, all patients presenting this mutation were tested for V460V (9). When $\mathrm{V} 460 \mathrm{~V}$ was detected, indicating the presence of a REC, mutations 55del and D409H were screened in order to determine the extent of the recombination sequence. Moreover, when a REC was detected, the patient's parents were tested, when possible, to determine if the mutations were in the same allele. The most frequent RECs are RecNciI (L444P, A456P and V460V) and RecTL (D409H, L444P, A456P, and V460V). Due to the absence of analysis of A456P, we opted to present RECs by the mutations actually detected. Finally, the 15 patients classified as types 2 and 3 were also tested for 55del, D409H and E326K due to the high frequency of these mutations among them.

\section{Results}

The sample analyzed was composed of $247(94 \%)$ type 1, $3(1 \%)$ type 2 and $12(5 \%)$ type 3 GD patients. Type 2 GD patients may be under-represented since they are not treated by ERT and have a short life expectancy. Nevertheless, the distribution was similar to that of other studies in distinct populations (1).

The genotypic distribution in the 247 type 1 patients is shown in Table 2. Among 
the first one hundred patients analyzed, only one allele harboring mutation 84insG and none with IVS2+1G $>$ A were detected. Thereafter, screening for these mutations was halted and they were considered to be unidentified. Almost half of the patients were compound heterozygotes presenting the N370S/L444P genotype. Combination of N370S with a second unidentified mutation was the second most frequent genotype, present in approximately $30 \%$ of the alleles. The remaining patients had many other genotypes.

Molecular analysis of 6 children with a clinical diagnosis of type 1 GD showed a genotype characteristic of type 3 GD (homozygous for L444P). For statistical purposes these children were considered to be type 1 patients according to their clinical characterization, although their genotypes suggest that neuronopathic symptoms may be expected to occur later in life.

The mutation frequencies, independently of their genotypic distribution, are presented as the first column of Table 3. While almost half of the patients' alleles carry N370S (47\%), the other half is divided between L444P (27\%), G377S (2\%) and an

Table 1. Screening target, primers and restriction enzymes used for the detection of some mutations that cause Gaucher disease.

\begin{tabular}{|c|c|c|c|c|}
\hline $\begin{array}{l}\text { Mutation } \\
\text { (cDNA sequence) }\end{array}$ & $\begin{array}{l}\text { Phenotypic } \\
\text { effect }^{\mathrm{a}}\end{array}$ & $\begin{array}{l}\text { Screening } \\
\text { target }\end{array}$ & F and $R$ primers (Ref.) & $\begin{array}{c}\text { Restriction } \\
\text { endonuclease }\end{array}$ \\
\hline N370S (c.1226A>G) & Mild & All patients & $\begin{array}{l}\text { F: 5'gcctttgtccttaccctcg3' } \\
\text { R: 5'acgaaagttacgcacccaatt3' (32) }\end{array}$ & Xhol \\
\hline G377S (c.1246G>A) & Mild/severe & All patients & $\begin{array}{l}\text { F: 5'gcctttgtccttaccctcg3' } \\
\text { R: 5'acgaaagttacgcacccaatt3' }\end{array}$ & Alul or Pvull \\
\hline L444P (c.1448T>C) & Severe & All patients & $\begin{array}{l}\text { F: 5'ctgaaccccgaaggaggac3' } \\
\text { R: 5'tgaatggagtagccaggtga3' (33) }\end{array}$ & Ncil \\
\hline V460V (c.1497G>C) & $\mathrm{b}$ & Patients with L444P & $\begin{array}{l}\text { F: 5'ctgaaccccgaaggaggac3' } \\
\text { R: 5'tgaatggagtagccaggtga3' }\end{array}$ & $B c g \mid$ \\
\hline 84insG (c.84insG) & Null allele & First 100 patients & $\begin{array}{l}\text { F: 5'cactgcctgaagtagatgc } 3^{\prime} \\
\text { R: 5'gaatgtcccaagcctttga3' }\end{array}$ & BsaBI \\
\hline IVS2+1G>A & Null allele & First 100 patients & $\begin{array}{l}\text { F: 5'gaatgtcccaagcctttga3' } \\
\text { R: 5'aacagagtaagactctggttc3' }\end{array}$ & $H p h l$ \\
\hline $\mathrm{D} 409 \mathrm{H}(\mathrm{c} .1342 \mathrm{G}>\mathrm{C})$ & Severe & $\begin{array}{c}\text { Types } 2 \text { and } 3 \\
\text { Patients with V460V }\end{array}$ & $\begin{array}{l}\text { F: 5'aaccatgattccctatcttc3' } \\
\text { R: 5'gctccctcgtggtgtagagt3' }\end{array}$ & Styl \\
\hline E326K (c.1093G>A) & c & Types 2 and 3 & $\begin{array}{l}\text { First PCR: } \\
\text { F: 5'acaaattagctgggtgtggc3' } \\
\text { R: 5'taagctcacactggccctgc3' } \\
\text { Nested PCR: } \\
\text { F: 5'tgtgcaaggtccaggatcag3' } \\
\text { R: 5'gaggtctgctttgcaggaag3' }\end{array}$ & Bbsl or Bpil \\
\hline $\begin{array}{l}\text { 55del } \\
\text { (c.1263_1317del) }\end{array}$ & Null allele & $\begin{array}{l}\text { Types } 2 \text { and } 3 \\
\text { Homozygotes for } \\
\text { N370S, G377S } \\
\text { and L444P } \\
\text { Patients with V460V }\end{array}$ & $\begin{array}{l}\text { F: 5'aaccatgattccctatcttc3' } \\
\text { R: 5'gctccctcgtggtgtagagt3' (8) }\end{array}$ & $d$ \\
\hline
\end{tabular}

aPhenotypic effect of mutations according to Ref. 3. Mutation G377S has been detected with both mild and severe effects (see text). ${ }^{b}$ Mutation V460V is a silent alteration used to indicate the presence of a recombinant allele. 'E326K is a modifier that may be present in combination with other Gaucher disease-causing mutations. dIdentification of mutation $55 \mathrm{del}$ may be visualized directly after PCR. 
unidentified mutation (24\%).

Since mutation L444P may be present alone or as part of a REC, this possibility was always examined. Among type $1 \mathrm{GD}$ patients, $46 \%(62 / 134)$ of alleles with L444P were found to be RECs with L444P+V460V. Testing for $\mathrm{D} 409 \mathrm{H}$ followed the detection of V460V and only $2 \%$ (3/134) of alleles with L444P were positive, disclosing a REC with L444P+V460V+D409H. Mutation 55del was not detected in any of the RECs, suggesting that they probably had pseudogene-derived mutations restricted to exons 9 and 10 . However, other recombination events upstream from $55 \mathrm{del}$ may be present. When including type 2 and 3 patients in the statistical analysis, RECs were identified in 44\% (66/150) of the chromosomes bearing mutation L444P and in 25\% (66/262) of all GD patients.

The 15 patients with type 2 and 3 GD were screened for mutations N370S, G377S, L444P, V460V, 55del, D409H, and E326K. Table 4 presents the genotypes found. We detected a homozygote patient whose parents are first cousins presenting E326K in combination with L444P in both alleles with a type 2 clinical phenotype. The only compound heterozygote presenting L444P in combination with a REC also had type 2 GD. Among the type 3 patients, the presence of mutations N370S in one case and G377S in 5 cases was unexpected since these are generally considered to be mild mutations (3).

\section{Discussion}

\section{Frequency of the most common mutations}

Although $47 \%$ of all GD-causing mutations are N370S (Table 3), only 10\% of the patients are N370S/N370S homozygotes (Table 2), a frequency much lower than the $22 \%$ expected $\left(0.47^{2}\right)$. These data probably reflect the fact that many N370S homozygotes are not obtaining medical attention due to the absence or mildness of symptoms. The 24 homozygotes herein detected indicate a penetrance of $45 \%$ for this genotype $(0.10 / 0.22)$, a value that is close to that described for the Ashkenazi Jewish population (10) and for the Portuguese population (11). These cases indi-

Table 2. Genotypic frequency observed in 247 Brazilian patients with type 1 Gaucher disease.

\begin{tabular}{lc}
\hline Genotype & Number of patients (frequency) \\
\hline N370S/L444P & $112(45.3 \%)$ \\
N370S/? & $70(28.3 \%)$ \\
N370S/N370S & $24(9.7 \%)$ \\
?/? & $19(7.7 \%)$ \\
L444P/? & $9(3.6 \%)$ \\
L444P/L444P & $6(2.4 \%)$ \\
G377S/G377S & $4(1.6 \%)$ \\
G377S/N370S & $2(0.8 \%)$ \\
G377S/L444P & $1(0.4 \%)$ \\
Total & $247(100 \%)$ \\
\hline
\end{tabular}

$?=$ unidentified mutation .

\begin{tabular}{|c|c|c|c|}
\hline Mutation & $\begin{array}{l}\text { Brazil } \\
\text { Present study } \\
\text { N (\%) }\end{array}$ & $\begin{array}{c}\text { United States of America } \\
\text { Zhao and Grabowski, } 2002(12) \\
\text { N (\%) }\end{array}$ & $\begin{array}{c}\text { Portugal } \\
\text { Amaral et al., } 1996(13) \\
\mathrm{N}(\%)\end{array}$ \\
\hline N370S & 232 (47.0\%) & $183(43.7 \%)$ & 29 (53.7\%) \\
\hline L444P & $134(27.1 \%)$ & $107(25.5 \%)$ & 7 (13.0\%) \\
\hline G377S & $11(2.2 \%)$ & Not determined & $4(7.4 \%)$ \\
\hline Others & $117(23.7 \%)$ & $129(30.8 \%)$ & $14(25.9 \%)$ \\
\hline Total & 494 alleles $(100 \%)$ & 419 alleles (100\%) & 54 alleles $(100 \%)$ \\
\hline
\end{tabular}

Statistical analysis comparing the present study with the North American sample considered G377S to belong to the "others" category. 
cate that despite its Mendelian mode of inheritance, GD presents complex phenotypes.

The frequency of the two most common GD-causing mutations among Brazilian patients is similar to that described for other populations, where N370S and L444P represent roughly half and one quarter of the alleles, respectively (1). Mutations 84insG and IVS2+1G $>$ A were found to be too rare. In fact, these mutations have a high frequency among Jewish patients but not in the general population of most countries (1). The low detection rate of the 84insG and IVS $2+1 \mathrm{G}>\mathrm{A}$ in the present study may reflect the low frequency of the Jewish population $(<0.1 \%)$ in Brazil.

From Table 3 it is possible to observe that the frequency of the most common mutations in Brazilian type 1 GD patients detected in the present study is similar to that found in a sample of non-Jewish North Americans (12) $(\mathrm{P}=0.263)$. However, it is

Table 4. Genotypes of 15 neuronopathic Brazilian patients with Gaucher disease.

\begin{tabular}{ll}
\hline Genotype & Phenotype \\
\hline L444P+E326K/L444P+E326Ka,b & Type 2 \\
L444P+V460V/L444P & Type 2 \\
L444P+E326K/L444Pb & Type 2 \\
L444P/L444P & Type 3 \\
L444P/D409H & Type 3 \\
L444P/D409H & Type 3 \\
L444P+V460V/? & Type 3 \\
L444P+V460V/? & Type 3 \\
55del/? & Type 3 \\
L444P+E326K/G377S & Type 3 $/$ ME \\
L444P+E326K/G377S & Type 3 \\
L444P/G377S/E326KC & Type 3 \\
G377S/? & Type 3 \\
G377S/? & Type 3 \\
L444P+V460V/N370S & Type 3d/ME \\
\hline
\end{tabular}

$\mathrm{ME}=$ myoclonic epilepsy. aParents are first cous-

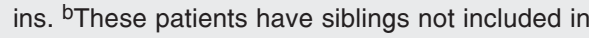
this study, presenting the same genotype and phenotype. 'E326K phase was not determined, i.e., it was cis or trans to L444P. dNeuronopathic patients with a clinical diagnosis of type 3 but carrying mutations considered to be mild. The patient carrying N370S did not have a detailed oculomotor evaluation. significantly different $(\mathrm{P}=0.028)$ when compared to that of Portuguese cases (13), according to chi-square analysis. The genetic background of the Brazilian population includes important African and Indigenous components, in addition to the Portuguese ancestry, that may explain the greater similarity to the North American sample.

Mutation G377S is the third most frequent among GD patients in Portugal and Spain (13). In Brazil, it also appeared at a considerable frequency $(2.2 \%)$, indicating that it is important in preliminary screening. A correspondence in mutations causing another lysosome storage disease has also been described for Tay-Sachs disease and points to a common origin of the mutated alleles prior to the Portuguese colonization of Brazil (14).

Among the alleles with L444P, 44\% (66/ 150 ) were shown to be RECs, present in $25.2 \%$ (66/262) of patients, corresponding to $12.6 \%$ $(66 / 524)$ of the alleles studied. Our approach was limited by the lack of analysis of the A456P mutation, present in the RECs. Therefore, we have opted to present RECs by the mutations actually diagnosed. Since some patients may have A456P and lack the silent mutation in V460V, the frequency of RECs in this sample may be even higher. The high frequency of RECs was also described in other countries $(9,15)$. It is generally observed that the RECs have a more severe impact on clinical manifestation than L444P alone (9,16-18). In agreement with this observation the three homozygotes for L444P additionally carrying E326K or RECs had type $2 \mathrm{GD}$, while those with genotype L444P/L444P but lacking complex alleles had milder phenotypes. It is possible that these patients' more severe phenotypes reflect a modifier effect of E326K and RECs leading to a lower residual enzyme activity, although other loci may also have influenced the severity of the phenotype $(4,19)$. The type 2 patient with the L444P+E326K/ L444P+E326K genotype did not present the severe perinatal-lethal phenotype, downplaying this genotype-phenotype correlation (20). 
Type 1 pre-neuronopathic affected children

Among type 3 GD patients, neurological manifestations appear after 10 years of age in $50 \%$ of the cases (1). Therefore, it is always possible that children under this age, diagnosed as type 1, may actually be type 3 patients that are in a pre-neuronopathic phase. Molecular diagnosis may help to identify these patients and this was the case for 6 children in this study, aged 5 to 6 years, in which mutation L444P was detected in homozygosity. It is possible that other children clinically classified as type 1 patients, with unidentified mutations and without a mild mutation, are in the same situation. Although only 5\% of GD patients are type 3 cases, the onset of GD is generally during infancy, which makes the possibility of a pre-neuronopathic patient a frequent doubt. The anticipation of the diagnosis of a neuronopathic GD variant by DNA testing is due to the need to inform the family, since these patients are likely to manifest developmental and language problems $(4,21)$. More important is the impact on ERT. One possibility is that a high-dose treatment may impact the neuronopathic symptoms $(21,22)$ but conclusive evidence is still lacking for GD. The European consensus for the treatment of neuronopathic GD has established that the ERT of type 3 patients, including the pre-neuronopathic ones identified by molecular diagnosis, is to be performed at a higher dose that should not be reduced even after symptoms ameliorate (23).

\section{Neuronopathic patients with mutations N370S and G377S}

The evidence for considering G377S a mild mutation is based on its high residual enzyme activity (17.6\%) according to measurement of acid B-glucosidase activity (24). Moreover, all reports of G377S homozygotes involve type 1 GD patients (16,25-27). Accordingly, in the present study four G377S homozygotes had a type 1 phenotype. How- ever, five compound heterozygotes carrying one G377S allele were type 3 patients. Other reports have also detected type 3 patients carrying one copy of G377S (3,28-30). These observations suggest the possibility of an allele-dose effect, whereby two copies of G377S would lead to an enzymatic residual activity sufficient to prevent neurological involvement, but one copy of G377S with a second severe mutation would lead to type 3 GD. An allele dose-effect has already been described for mutation K79N (31). In this model, the residual activity of mutant enzymes produced by the two alleles should be considered in an additive manner. Some mutations, such as G377S, K79N, N188S, V394L, and R463C could be at the limit of residual activity that elicits neuropathic symptoms $(3,16,31)$.

The patients reported here represent further in vivo evidence for a critical role of a GD-causing mutation in the development of neurological disease. However, the participation of other modifiers cannot be excluded. In this study, another Brazilian family with two siblings (aged 5 and 15 years) presenting the G377S/L444P genotype lacked any neurological involvement (Table 2). These siblings were shown not to carry the E326K mutation (data not shown), while 2 other patients with genotype G377S/L444P+ E326K presented neuronopathic symptoms (Table 4). This finding reinforces a possible negative modifier effect of E326K.

The last case in Table 4 presents neuronopathic symptoms that appeared to be inconsistent with his genotype (N370S/ L444P+V460V). Mutation N370S is in trans to the REC allele that was inherited from the mother. The mild effect of N370S has been well described in hundreds of type 1 GD patients reported so far and contrasts to the neuronopathic phenotype in this case. However, the patient herein described may suffer from hypoxic-ischemic encephalopathy and therefore her neuronopathic manifestation may not be a consequence of GD. 
It is clear that DNA testing provides information both on the diagnosis and the prognosis of GD and new insights on genotype-phenotype correlations should be promptly integrated into medical practice. Moreover, since ERT is individualized and the treatment dose is subject to medical decision, molecular diagnosis may be critical to establish a proper dose, as in the case of the L444P homozygote children still lacking neurological symptoms. Finally, our findings indicate that an allele dose-effect may be associated with mutation G377S. This mutation should be part of the screening panel for GD patients from Iberian countries, including their former colonies. Further studies may be critical to establish its exact genotype-phenotype correlation.

\section{Acknowledgments}

The authors wish to thank the following persons for their valuable contributions: Dr.
Angela Morgante, Dr. Fernanda Teresa de Lima, Katia Maria da Rocha, Dr. Martha Lima, Dr. Paulo Alberto Otto, and Dr. Regina Celia Mingroni Neto from IB-USP; Dr. Gilda Porta from FM-USP; Dr. Ana Paula Constancio, Andrea Callipo, Dr. Carlos Ruchaud, Daniella Jorge, Rodrigo Azambuja, Cristiana Petriz, and Dr. Rogerio Vivaldi from Genzyme do Brasil; Dr. Angela Zanette from HEMOBA; Dr. Cintia Xerez from HEMOAM; Dr. Maria Cristina Pereira, Dr. Maria Teresa M. de Paula, and Dr. Renata Cravo from HEMORIO; Dr. Silvia Thomas from HEMOMAT; Dr. Cristina Bortolheiro and Dr. Cristina Pozzi from Santa Casa de Misericórdia SP; Dr. Erlane Ribeiro from Hospital Albert Sabin CE; Dr. Eugenia Ribeiro Valadares from HC-BH; Lara Franco from UH; Dr. Mara A.D. Pianovski; Dr. Marcelo Kerstenetzki from Hospital de Restauração (PE), and Dr. Maria de Fatima Montoril from HEMOPA.

\section{References}

1. Beutler E, Grabowski GA. Gaucher disease. In: Scriver CR, Beaudet AL, Sly WS, Valle DV (Editors), The metabolic and molecular bases of inherited disease. 8th edn. New York: McGraw-Hill; 2001. p 36353668.

2. Sorge J, West C, Westwood B, Beutler E. Molecular cloning and nucleotide sequence of human glucocerebrosidase cDNA. Proc Natl Acad Sci U S A 1985; 82: 7289-7293.

3. Beutler E, Gelbart T, Scott CR. Hematologically important mutations: Gaucher disease. Blood Cells Mol Dis 2005; 35: 355-364.

4. Goker-Alpan O, Hruska KS, Orvisky E, Kishnani PS, Stubblefield BK, Schiffmann R, et al. Divergent phenotypes in Gaucher disease implicate the role of modifiers. J Med Genet 2005; 42: e37.

5. Germain DP. Gaucher's disease: a paradigm for interventional genetics. Clin Genet 2004; 65: 77-86.

6. Michelin K, Wajner A, de Souza FT, de Mello AS, Burin MG, Pereira $\mathrm{ML}$, et al. Application of a comprehensive protocol for the identification of Gaucher disease in Brazil. Am J Med Genet A 2005; 136: 5862.

7. Richards B, Skoletsky J, Shuber AP, Balfour R, Stern RC, Dorkin $\mathrm{HL}$, et al. Multiplex PCR amplification from the CFTR gene using DNA prepared from buccal brushes/swabs. Hum Mol Genet 1993; 2: 159-163.

8. Tayebi N, Stern H, Dymarskaia I, Herman J, Sidransky E. 55-Base pair deletion in certain patients with Gaucher disease complicates screening for common Gaucher alleles. Am J Med Genet 1996; 66: 316-319.
9. Tayebi N, Stubblefield BK, Park JK, Orvisky E, Walker JM, Lamarca $M E$, et al. Reciprocal and nonreciprocal recombination at the glucocerebrosidase gene region: implications for complexity in Gaucher disease. Am J Hum Genet 2003; 72: 519-534.

10. Beutler E, Nguyen NJ, Henneberger MW, Smolec JM, McPherson RA, West $C$, et al. Gaucher disease: gene frequencies in the Ashkenazi Jewish population. Am J Hum Genet 1993; 52: 85-88.

11. Lacerda L, Amaral O, Pinto R, Oliveira P, Aerts J, Sa Miranda MC. Gaucher disease: N370S glucocerebrosidase gene frequency in the Portuguese population. Clin Genet 1994; 45: 298-300.

12. Zhao H, Grabowski GA. Gaucher disease: Perspectives on a prototype lysosomal disease. Cell Mol Life Sci 2002; 59: 694-707.

13. Amaral O, Pinto E, Fortuna M, Lacerda L, Sa Miranda MC. Type 1 Gaucher disease: identification of N396T and prevalence of glucocerebrosidase mutations in the Portuguese. Hum Mutat 1996; 8: $280-281$.

14. Rozenberg R, Martins AM, Micheletti C, Mustacchi Z, Pereira LV. Tay-Sachs disease in Brazilian patients: prevalence of the IVS7+1g>c mutation. J Inherit Metab Dis 2004; 27: 109-110.

15. Cormand B, Harboe TL, Gort L, Campoy C, Blanco M, Chamoles N, et al. Mutation analysis of Gaucher disease patients from Argentina: high prevalence of the RecNcil mutation. Am J Med Genet 1998; 80: 343-351.

16. Koprivica V, Stone DL, Park JK, Callahan M, Frisch A, Cohen IJ, et al. Analysis and classification of 304 mutant alleles in patients with type 1 and type 3 Gaucher disease. Am J Hum Genet 2000; 66: 
1777-1786.

17. Stone DL, Tayebi N, Orvisky E, Stubblefield B, Madike V, Sidransky E. Glucocerebrosidase gene mutations in patients with type 2 Gaucher disease. Hum Mutat 2000; 15: 181-188.

18. Mignot C, Gelot A, Bessieres B, Daffos F, Voyer M, Menez F, et al. Perinatal-lethal Gaucher disease. Am J Med Genet A 2003; 120 : 338-344.

19. Montfort M, Chabas A, Vilageliu L, Grinberg D. Functional analysis of 13 GBA mutant alleles identified in Gaucher disease patients: Pathogenic changes and "modifier" polymorphisms. Hum Mutat 2004; 23: 567-575.

20. Chabas A, Gort L, Diaz-Font A, Montfort M, Santamaria R, Cidras M, et al. Perinatal lethal phenotype with generalized ichthyosis in a type 2 Gaucher disease patient with the [L444P;E326K]/P182L genotype: effect of the E326K change in neonatal and classic forms of the disease. Blood Cells Mol Dis 2005; 35: 253-258.

21. Erikson A, Astrom M, Mansson JE. Enzyme infusion therapy of the Norrbottnian (type 3) Gaucher disease. Neuropediatrics 1995; 26 : 203-207.

22. Vogler C, Levy B, Grubb JH, Galvin N, Tan Y, Kakkis E, et al. Overcoming the blood-brain barrier with high-dose enzyme replacement therapy in murine mucopolysaccharidosis VII. Proc Natl Acad Sci U S A 2005; 102: 14777-14782.

23. Vellodi A, Bembi B, de Villemeur TB, Collin-Histed T, Erikson A, Mengel E, et al. Management of neuronopathic Gaucher disease: a European consensus. J Inherit Metab Dis 2001; 24: 319-327.

24. Amaral O, Marcao A, Sa MM, Desnick RJ, Grace ME. Gaucher disease: expression and characterization of mild and severe acid beta-glucosidase mutations in Portuguese type 1 patients. Eur $J$ Hum Genet 2000; 8: 95-102.
25. Amaral O, Lacerda L, Marcao A, Pinto E, Tamagnini G, Sa Miranda MC. Homozygosity for two mild glucocerebrosidase mutations of probable Iberian origin. Clin Genet 1999; 56: 100-102.

26. Bembi B, Zambito MS, Sidransky E, Ciana G, Carrozzi M, Zorzon M, et al. Gaucher's disease with Parkinson's disease: clinical and pathological aspects. Neurology 2003; 61: 99-101.

27. Giraldo P, Pocovi M, Perez-Calvo J, Rubio-Felix D, Giralt M. Report of the Spanish Gaucher's disease registry: clinical and genetic characteristics. Haematologica 2000; 85: 792-799.

28. Germain DP, Kaneski CR, Brady RO. Mutation analysis of the acid beta-glucosidase gene in a patient with type 3 Gaucher disease and neutralizing antibody to alglucerase. Mutat Res 2001; 483: 89-94.

29. Park JK, Orvisky E, Tayebi N, Kaneski C, Lamarca ME, Stubblefield BK, et al. Myoclonic epilepsy in Gaucher disease: genotype-phenotype insights from a rare patient subgroup. Pediatr Res 2003; 53: 387-395.

30. Wong K, Sidransky E, Verma A, Mixon T, Sandberg GD, Wakefield LK, et al. Neuropathology provides clues to the pathophysiology of Gaucher disease. Mol Genet Metab 2004; 82: 192-207.

31. Zhao H, Bailey LA, Elsas LJ, Grinzaid KA, Grabowski GA. Gaucher disease: in vivo evidence for allele dose leading to neuronopathic and nonneuronopathic phenotypes. Am J Med Genet A 2003; 116 : 52-56.

32. Beutler E, Gelbart T, West C. The facile detection of the nt 1226 mutation of glucocerebrosidase by 'mismatched' PCR. Clin Chim Acta 1990; 194: 161-166.

33. Tsuji S, Choudary PV, Martin BM, Stubblefield BK, Mayor JA, Barranger JA, et al. A mutation in the human glucocerebrosidase gene in neuronopathic Gaucher's disease. N Engl J Med 1987; 316: 570-575. 


\section{David Rockefeller Center for Latin American Studies Harvard University}

\author{
"Lemann Visiting Scholars and Fellows \\ Program" Brasil
}

The David Rockefeller Center for Latin American Studies, Harvard University, invites candidates for its "Visiting Scholars and Fellows Program". The "Visiting Scholars and Fellows Program" is a program for academic and non-academic professionals interested in developing research for a determined period of time (one or two academic semesters) while residing at Harvard University. The selection of professionals is made by an examination.

With the support of Mr. Jorge Paulo Lemann, the Center recently created the "Lemann Visiting Fellowship". This is a fellowship for a professional for the above mentioned program whose research project has Brazil as the study objective. This fellowship is awarded once each academic year (September-June) and covers the administrative expenses of the Center and airline tickets. The professional will also receive financial assistance of US\$15,000 (fifteen thousand American dollars, subject to income tax), regardless of the number of semesters for which he is accepted.

\section{Candidates must include, in English:}

- Curriculum vitae;

- One page explaining why the candidate wishes to study at Harvard;

- Three or four pages describing the proposed research project;

- Two letters of reference from persons who can prove the qualifications of the candidate and the importance of the proposed project.

\section{For additional information, contact:}

Visiting Scholars and Fellows Program Coordinator David Rockefeller Center for Latin American Studies Harvard University

61 Kirkland Street

Cambridge, MA 02138 USA

Tel: (617) 495-3366 Fax: (617) 496-2802

e-mail:drclas@fas.harvard.edu

Web site: http://www.fas.harvard.edu/ drclas

\section{Post-doctorate fellowship in Germany Alexander Von Humboldt Foundation}

The Alexander Von Humboldt Foundation, which exists more than 130 years and whose actual structure was established a few years after WWII (1953), is offering fellowships for post-doctorate study and for specialized research projects in all areas of knowledge. There are 500 fellowships annually, distributed without limitation of nationality, and 150 fellowships (Feodor Lynen Research Fellowships), only for German researchers and professors, to work as invited researchers of ex-fellows of all nationalities and their Universities. The Alexander Von Humboldt Foundation Fellowships are open to all professors who meet the above-mentioned requisites and who are less than 40 years of age. There is also one specialized fellowship for candidates between 40 and 45 years of age. These fellowships are for 6 to 24 months, receiving 3400 to 4200 German marks monthly.

\author{
Interested candidates should contact: \\ Alexander Von Humboldt-Stiftung \\ Jean Paul Strasse 12 \\ D-53173 BONN (Germany) \\ Telefax: 0049-228-833-199 \\ Internet: http://www.avh.de
}

Ex-fellows of the Foundation are represented in Brazil by:

"Clube Humboldt do Brasil"

Av. Brig. Faria Lima, $1572-7^{\circ}$ andar, sala 705

01463-900 São Paulo, SP

President Prof. Dr. Bruno Konig Junior

Department of Anatomy, ICB, USP, São Paulo, Brasil

Tel: (011)818-7258 